$\xi=-1$

\title{
Video Transmission Technique through Dual Transmission of Visible Light / Non-Audible Sound Communication in VLC System
}

\author{
Doohee Han ${ }^{1}$, Kyujin Lee ${ }^{2 *}$ \\ ${ }^{1}$ Kyung Hee University, 1 Seocheon-dong, Giheung-gu, Yongin-si, Gyeonggi-do, 446-701, Republic of Korea \\ ${ }_{2}^{2}$ Professor, Department of Electronic Engineering, Semyung University, 65 Semyung-ro, Jecheon-siChungcheongbuk-do, 27136, \\ Republic of Korea \\ *Corresponding author E-mail: kyujin@semyung.ac.kr
}

\begin{abstract}
Background/Objectives: In this paper, we study the video transmission technique through dual transmission technique which uses visible light communication and non - audible sound communication simultaneously in visible light communication system.

Methods/Statistical analysis: In high-speed video and multimedia transmission environments, existing visible light communication(VLC) has a problem in that communication is disconnected when visible light does not reach directly or when a shadow area occurs due to an obstacle. Unlike RF communication, if the line of sight due to direct light is not secured, the communication performance deteriorates sharply. In order to solve such a problem, the non-audible sound communication is transmitted in dual with visible light communication so that communication disruption does not occur when a shadow region due to an obstacle occurs. We also proposed a scheme to improve the overall system through diversity gain.

Findings: We proposed the video transmission method through dual transmission technique to improve the data rate and quality via VLC and sound communication. This solves the problem of shadowing of existing visible light communication. We expect that the performance of the entire system will be improved through diversity gain through dual transmission.

Improvements/Applications: The dual transmission technique which uses visible light communication and non - audible sound communication system can be applied as fusion technology to various environments such as an IoT network environment requiring a bidirectional network or an indoor small-scale network configuration and a home network configuration.
\end{abstract}

Keywords: VLC System, Dual transmission, non-audible sound wave communication, Shadowing, fusion technology

\section{Introduction}

Recently, the LED related market is attracting attention as the green industry and next generation information communication technology. Compared to conventional fluorescent lamps and gas lamps, they are attracting attention as eco-friendly devices because they emit less environmental pollutants and can use low power consumption, long life, and harmless visible light sources selectively. Visible Light Communication (VLC) systems are convergence technologies that provide simultaneous illumination and data transmission through LEDs. The wavelength of light is different from optical communication technology through existing wired optical fiber by transmitting and exchanging information by using light including visible light region and near infrared region visible to human eyes. It is also possible to implement various colors. Generation communication system in which information is transmitted to each object and reused using an infrastructure in which light such as an incandescent lamp and a fluorescent lamp is replaced with a light emitting diode (LED) light. In addition, the wide bandwidth (THz) of the LED light source enables high-speed data transmission compared to conventional RF communication in designing a wireless optical communication system.[1][2] However, existing visible light communication has a problem in that communication is disconnected when visible light does not reach directly or when a shadow area occurs due to an obstacle. Unlike RF communication, if the line of sight due to direct light is not secured, the communication performance deteriorates sharply. Especially, in case of voice or text data, it is possible to restore by analogy, but in case of video signal, it is difficult. In order to overcome the limitation of visible light communication, we proposed dual transmission method using non - audible sound wave communication. Acoustic communication has the advantage that it has stronger characteristics in the shaded region than the optical based signal and is more flexible than the optical signal. In addition, the system can be configured with a simple structure using a general audio interface, which is strong against signal distortion and relatively simple in structure. However, since the transmission speed of sound wave communication is slow, it is difficult to transmit high quality video data. Therefore, we propose a technique to prevent communication failure in shadow areas through dual transmission of visible light communication and acoustic wave communication, and to satisfy QoS by seamlessly transmitting high quality video data. 


\section{System Model}

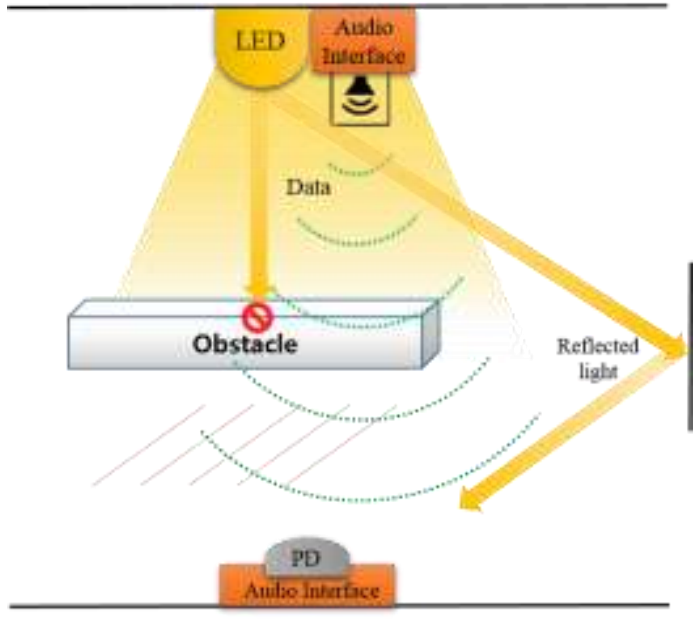

Fig. 1: Dual transmission system model

Figure 1 shows a model example of the proposed system in LOS(Line of Sight) environment. Due to the nature of visible light communication, data discontinuity occurs instantaneously when shadowing due to obstacles occurs. Existing systems suffer severe performance degradation in such environments. In the proposed system, it is possible to minimize the data disconnection even if momentary data discontinuity due to shadowing occurs due to low speed data switching through non-audible sound wave communication. Accordingly, a technique for solving such a shadowing problem and allowing video data to be transmitted seamlessly has been proposed.

\subsection{Channel Model}

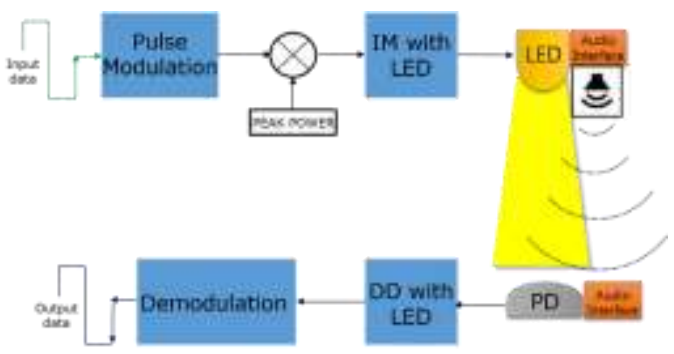

Fig. 2: VLC system structure

The system is a LOS (Line of Sight) link environment. The VLC system structure is shown in Fig. 2 The modulation method uses IM / DD. IM / DD modulation is a modulation scheme that changes the bias current of the LED. The receiver's photodetector produces a photocurrent proportional to the optical output of the received signal. VLC systems operate in the visible light and wavelength bands. The channel model is shown in the following equation. [3] The transmitted waveform is the instantaneous optical power of the wave emitter. The received waveform is proportional to the instantaneous power received by the instantaneous current of the received photodetector (PD). The channel noise is an additive white gaussian noise (AWGN) environment. [4]

$\mathrm{R}(\mathrm{t})=\alpha \mathrm{S}(\mathrm{t}) * \mathrm{G}(\mathrm{t})+\mathrm{N}(\mathrm{t})$

where the $*$ symbol denote convolution and is the detector responsively. $\mathrm{G}(\mathrm{t})$ is the impulse response, $\mathrm{N}(\mathrm{t})$ represents the AWGN.

The position of the transmitter is represented by $\mathrm{H}=\left(r_{s}, n_{s}, n\right)$ (position vector $r_{s}$, direction vector $n_{s}$, mode number of radiation lobe $\mathrm{n}$ ), $\mathrm{K}=\left\{r_{D}, \hat{n}_{D}, A_{D}, F O V\right\}$ (position vector $r_{D}$, direction vector $\hat{n}_{D}$, receiving area $A_{D}$, field of view $\left.(\mathrm{FOV})\right)$. In an indoor environment with a reflective surface, the channel impulse response can be expressed as:

$$
\mathrm{G}(\mathrm{t} ; \mathrm{H}, \mathrm{K})=\sum_{k=1}^{\infty} G^{k}(t ; H, \mathrm{~K})
$$

Here, $G^{(k)}(t)$ represents the impulse response of the signal that is reflected $\mathrm{k}$ times. The high-order terms of the channel impulse response considering the LOS signal are expressed as follows.

$\mathrm{G}(\mathrm{t} ; \mathrm{H}, \mathrm{K})=\int G^{(0)}\left(t ; H,\left\{r, \hat{n}, \frac{\pi}{2}, d r^{2}\right\} * G^{(k-1)}(t ;\{r, \widehat{n}, 1\}, K)\right.$

Here, $r$ represents the position vector on all the reflection surfaces S. $\hat{n}$ represents the unit normal vector at the position $r$ in the reflection plane $\mathrm{H}$, and $\mathrm{d} r^{2}$ is the diffraction plane at the positionr of the reflection surface.[5]

\subsection{Non-Audible Sound Communication}

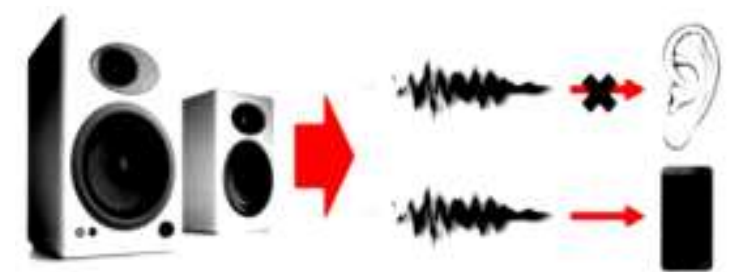

Fig. 3: Features of non-audible sonic communication.[6]

Figure.3 shows the Features of the Non-audible sound communication. Sound wave communication is a communication technology that transmits information by using a sound wave as a medium, and it is divided into an underwater sound communication and a standby sound wave communication depending on the kind of a medium in which sound is propagated. The sound wave has the characteristics that the propagation speed is faster and the transmission range is larger in the medium of high density. Therefore, in the past, research has focused on underwater sound communication such as underwater sonar detection technology and marine ecosystem monitoring sensor network. However, as interest in the sound communication in the air utilizing the built-in audio interface of smart devices has increased recently, related researches are actively being carried out. In the air, sound wave communication is a technique of communicating by placing information on sound waves in the air. Compared to underwater sonar communication using water, the transmission distance is as much as 10 meters because of the large loss of sound waves in the air. For this reason, different transmitters can be used depending on the communication operating frequency. Ultrasonic transceivers are used for $22 \mathrm{kHz}$ or more, and general audio interfaces for reproducing and recording audio for $22 \mathrm{kHz}$ or less. [6]

\subsection{Channel Model of Non-Audible Sound Communication}

Most microphones in our audio and smart devices that we have easy access to can usually play or record frequencies up to 22.05 $\mathrm{kHz}$. However, the inherent characteristics of the frequency response of these devices are different. In the case of a microphone built in a general speaker or a smart device, communication performance in a non-audible band is relatively low because the microphone is optimized for components up to the audible range. In the case of the PSK signal, the transmission efficiency of the signal is very good, and it is mainly used in the mobile communication fields such as CDMA and LTE. However, unlike the mobile communication field, in the case of the sound wave communication, since the frequency band of the signal uses a frequency band relatively lower than that of the mobile communication field, the possibility of signal distortion is 
considerably high. On the other hand, the chirp signal has a merit that it is simple in structure due to the technology based on the analog circuit, so the throughput is relatively low and the power consumption is not high.

Chirp signal is a signal widely used in RADAR communication because of its ability to separate multiple receiving components by multipath propagation. The chirp signal has a feature of sweeping not only one frequency component but also a specific frequency band. This chirp signal, also referred to as a linear filter, is referred to as an up-chirp when the frequency linearly increases and a down-chirp when the frequency linearly decreases. The expression of this linear chirp signal is as follows.[7][8]

$S_{1}(t)=\cos \left(2 \pi f_{L} t+\frac{\mu t^{2}}{2}\right) 0 \leq t \leq T$
$S_{2}(t)=\cos \left(2 \pi f_{H} t+\frac{\mu t^{2}}{2}\right) 0 \leq t \leq T$

Here, $S_{1}(t)$ is the up chirp and $S_{2}(t)$ is the down chirp equation. The expression of $\mu$, which means the amount of frequency change, is as follows.

$\mu=\frac{2 \pi\left(f_{H}-f_{L}\right)}{T}=\frac{2 \pi B}{T}$

Where $\mathrm{B}$ the frequency is band and $\mathrm{T}$ is the duration of the symbol. $f_{L}$ Means the lowest frequency in the frequency band, and $f_{H}$ means the highest frequency in the frequency band.

\subsection{Scalable Video Coding}

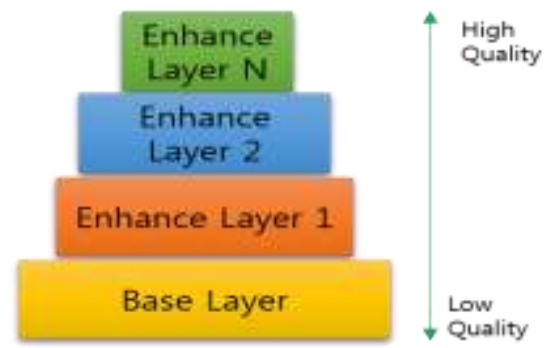

Fig. 4: Scalable Video Coding Conceptual diagram

Figure.4 shows the conceptual diagram of the Scalable Video Coding (SVC). SVC is a kind of H.264 / MPEG4-AVC standard video codec for time-varying channels. The SVC technique enables a high level of video bitstream coding. SVC signals are divided into layered coded signals in two group hierarchies. One is the SVC signal with high priority in the video image with the base layer sequence. The other is an enhancement layer sequence that is combined with the base layer sequence data to improve the QoS of the received video. These two layers of signal have different significance. In case of base layer signal, lower BER performance should be satisfied to provide minimum video service, and enhancement layer signal is required to improve BER performance because it is data for improving video quality. Thus, the BER requirements of the SVC layer differ from layer to layer. If a loss occurs in the transmission of the base layer signal, the first enhancement layer signal can not be received because the base layer signal information is needed at the receiving end when decoding the first enhancement layer signal. In other words, the base layer must be lower than the enhancement layer, and at the same time the protection order is reduced from level $n$ to level 0 when bandwidth and other conditions are restricted. Abstractly, if you get the best protection for both the base layer signal and the enhancement layer signal, you will get optimal system PSNR performance. The quality of the received Scalable video signal is as follows.[9][10]

$Q_{L}=\sum_{l=1}^{L} P(l) \cdot P S N R_{l}$

\section{Visible Light / Non-Audible Sound Wave Dual Transmission}

\subsection{The Proposed System Model}

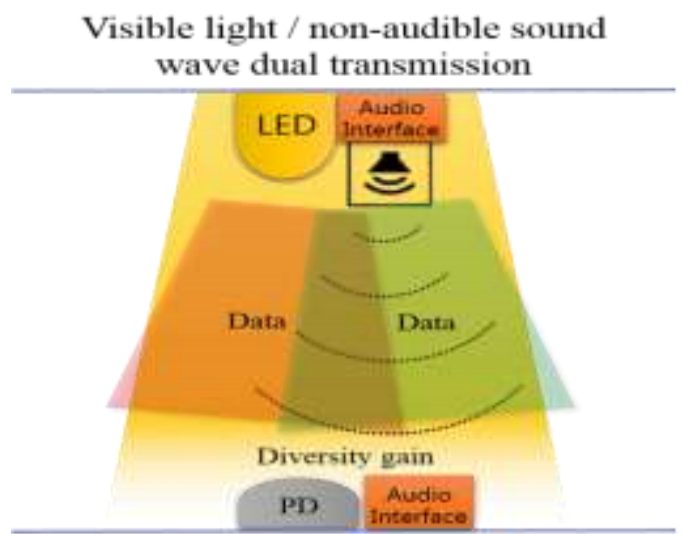

Fig. 5: Visible light / non-audible sound wave dual transmission model

In this paper, we propose a dual transmission system which combines high speed VLC communication system and acoustic wave communication of non - audible spectrum. It is a heterogeneous network convergence technology that overcomes the limitation of existing single link VLC system and is optimized for multimedia service. The proposed system implements the OnOff Keying (OOK) with combining Sound communication and LED. Figure. 5Shows the proposed system structure. The relatively slow sound wave communication continuously transmits the base layer which is the most important layer of the SVC video signal. Also, a fast VLC system transmits base layer and enhance layer simultaneously. With this dual transmission technique, it is possible to transmit SVC video data stably even if shadow areas occur due to difficulty in obtaining LOS.

Also, one of the advantages of visible light communication is that it is free from RF interference. By using sound communication, the advantage of VLC can be maintained. In the VLC, the LOS between the transmitter and the receiver must be secured at all times, and the proposed technique can overcome this limitation.

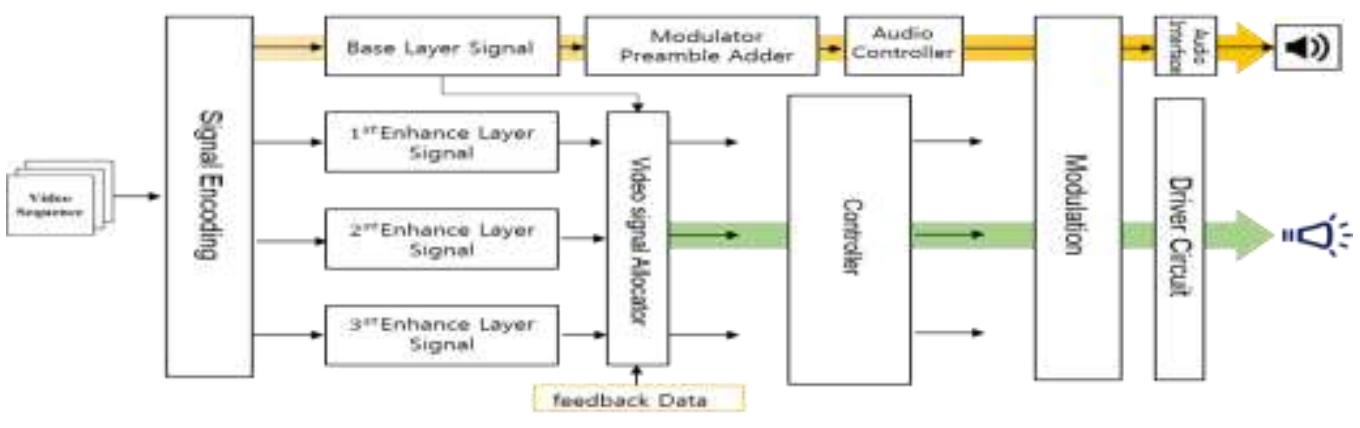




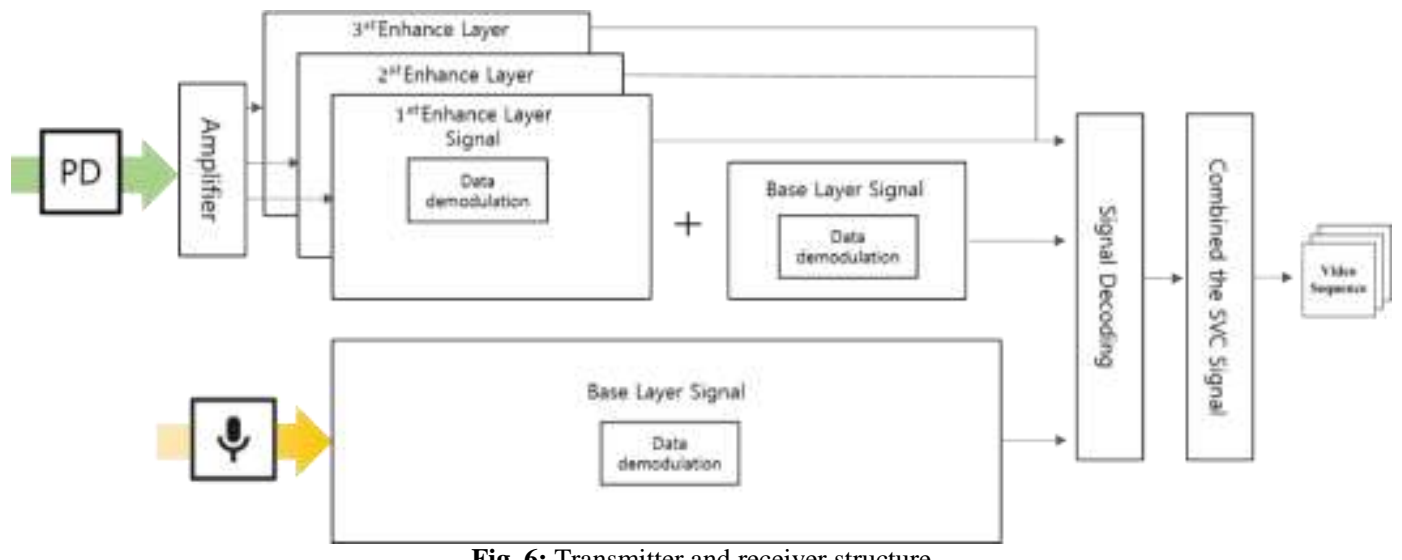

Fig. 6: Transmitter and receiver structure

Figure.6 shows the structure of the system transmitter and receiver. In the SVC video signal, the most important base layer is dual-transmitted by LED and sound wave communication to obtain the diversity gain. Even if a shadowing phenomenon occurs due to an obstruction of the LED by the obstacle, the base layer is continuously transmitted through the sound wave communication, thereby preventing the data from being disconnected. Also, the enhancement layers are simultaneously transmitted to LEDs with relatively fast transmission speeds, and the received signals are combined with each other at the receiving end to reconstruct the video signal

\section{Case 1: Dual Reception of VLC and Audio Signals}

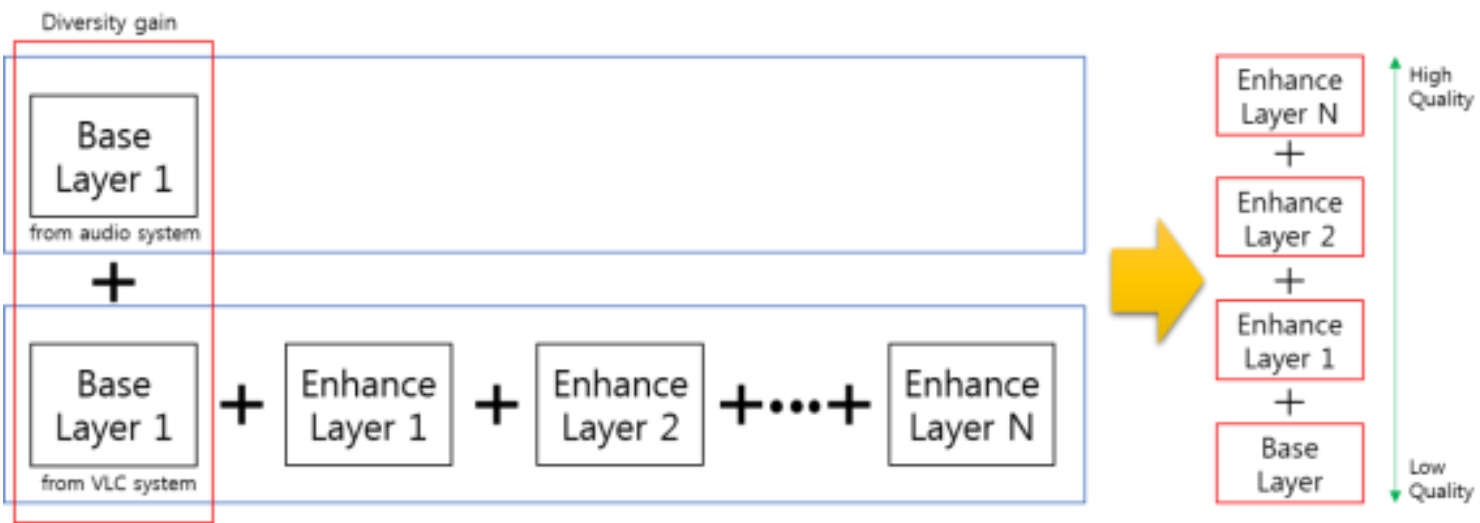

Case 2: Only audio signal received because the VLC system are blocked by obstacles.

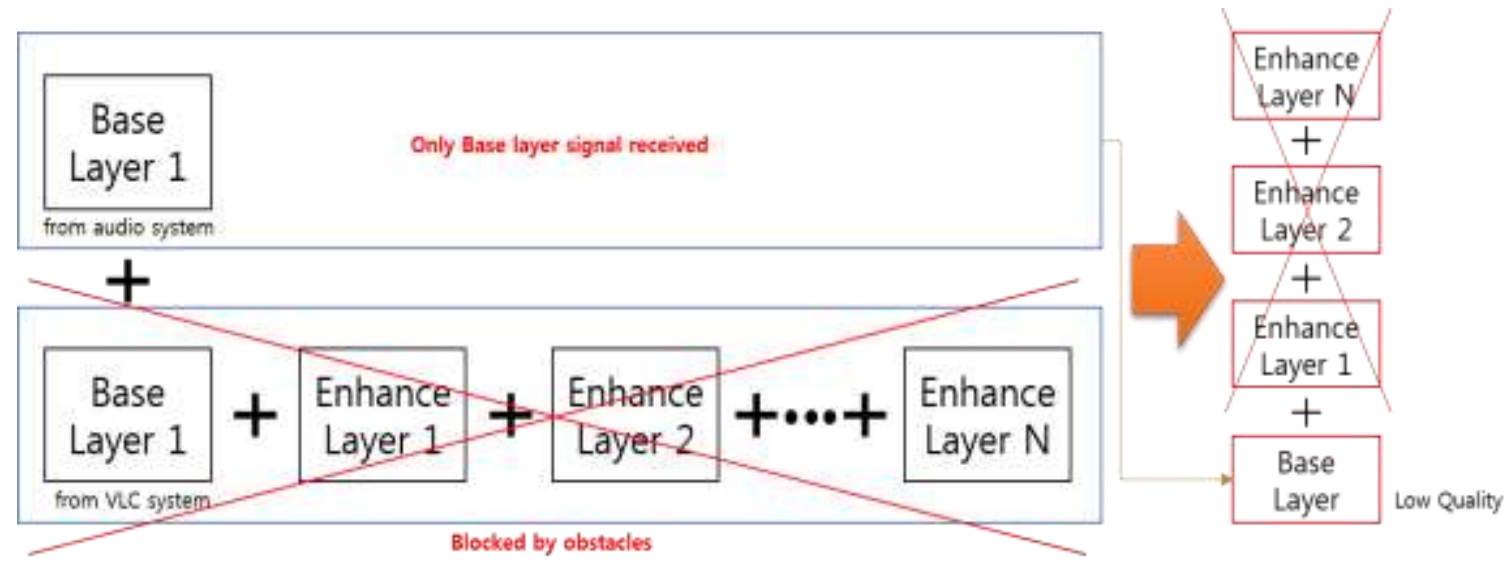

Fig 7: System structure

Figure.7 shows the overall system structure. As shown in Case 1, the sound wave communication continues to transmit the base layer. In the VLC system, the base layer and enhance layer are allocated to the respective channels and transmitted at the same time. At this time, the diversity gain due to the difference in the delay rate between the base layer of the low-speed sound communication and the base layer of the VLC system of the highspeed transmission is obtained, and the high-speed enhancement layer is received and synthesized to improve the QoS of the entire system. However, when an obstacle is generated between the LED transmitter and the receiver and the shadowing phenomenon occurs as in Case 2, it is difficult to transmit and receive data reliably from the VLC system. However, sound wave communication can communicate relatively freely from obstacles. Since the most important signal in the SVC video signal is the base layer, even if the enhance layer is partially abandoned, the signal can be reproduced if the base layer is secured. Therefore, even if the vlc system is temporarily shadowed, it can still transmit base layer through sonic communication to prevent data loss and maintain minimum video quality. 


\subsection{Simulation Results}



Fig. 8: PSNR performance in single transmission of sound communication due to shaded area occurrence

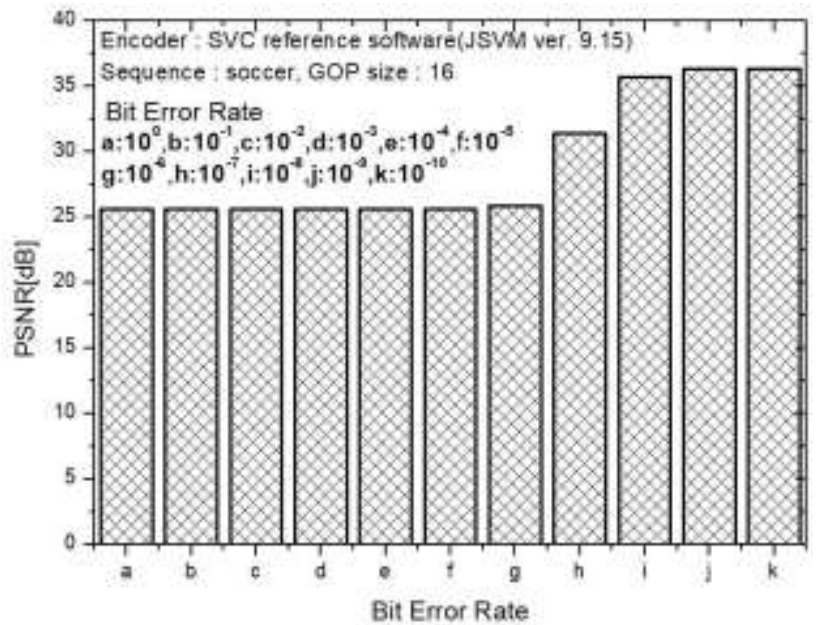

Fig. 9: PSNR performance of signals received simultaneously through VLC and sound communication dual transmission

Figure. 8 and 9 show the simulation results. Data transmission rate was $400 \mathrm{Mbps}$, AWGN noise model and background light noise were $0[\mathrm{dBm}]$. The scalable video stream is Joint Scalable Video Model (JSVM) ver. 13.1, Base layer, Enhance layer 1, and Enhance 2, and they are transmitted through the sound wave communication and the VLC. Figure 8 shows the case where the base layer and the enhancement layer of the LED transmitter are blocked due to the shadowing due to the obstacle. Therefore, it can be seen that the initial PSNR performance is drastically reduced. However, since the base layer is continuously transmitted at a low speed through the sound wave communication, the PSNR performance is gradually recovered even in the state where the shadowing is continued, so that the minimum video quality is maintained in the g part. Figure 9 shows that the base layer is simultaneously transmitted through dual transmission to obtain the diversity gain and the optimal PSNR performance through stable reception of the enhancement layer. It can be seen that the dual transmission improves performance over the existing one channel transmission system.

\section{Conclusion}

Advantages of visible light communication are as follows.

1. Ensure free communication in RF-free environment

2. Excellent security due to limited local area communication

The use of an RF system eliminates the advantages of such VLC.

Therefore, in this paper, we propose dual transmission of non- audible sound and visible light communication for stable video data transmission while resolving the shadow region while maintaining these advantages in visible light communication system. Through the proposed method can solve system performance degradation due to instantaneous shadowing. We also expect that the diversity gain will improve the performance of the system. However, since the data transmission speed of nonaudible sound wave communication is very low, research is needed to solve this problem. In order to construct a system for transmitting a large amount of data in the future, researches for solving low transmission rate problems should be supplemented.

\section{Acknowledgment}

This work was supported by the National Research Foundation of Korea(NRF) grant funded by the Korea government(MSIT) (No. 2017R1C1B5017812)

\section{References}

[1] H. Sugiyama, S. Haruyama, and M. Nakagawa, "Experimental investigation of modulation method for visible-light communications,'IEICE Trans. Commun., vol. E89-B, no. 12, pp. 3393-3400, Dec. 2006.

[2] D. G. Kim (2014. 01). Current status and direction of $5 \mathrm{G}$ mobile communication R\&D Information and Communication, 23-28.

[3] D.C.O'Brien et al, " Visible light communication: state of the art and prospects," published in Proc. Wireless World Research Forum 2007.

[4] Kyuntak Kim, Kyujin Lee "Performance Evaluation and Analysis of Zero Reduction Codes for Effective Dimming Control in Optical Wireless Communications using LED Lightings" Journal of Convergence for Information Technology Vol. 7 No.3 pp.97-103

[5] M.Z.Afgani, H.Haas, H.Elgala, D.Knipp, "Visible light communication using OFDM," Proc. IEEE Symp. on Wire- less Pervasive Computing, TRIDENTCOM 2006

[6] Su-Cheol Shin, Tae Hyun Kim, Jun-Won Choi "Inaudible Bandbased Aerial Sound Communication Systemfor T-Commerce" Proceedings of Symposium of the Korean Institute of communications and Information Sciences , 2015.06,838-839 (2 pages)

[7] K.Liu, X.Liu, and X.Li, "Acoustic Ranging and Communication via Microphone Channel," in proc.Global Communications Conf. (GLOBECOM),Anaheim, U.S.A., Dec. 2012.

[8] H.Lee, T.Kim, J.Choi, and S.Choi, "ChirpSignal-Based Aerial Acoustic Communication for Smart Devices," 2015 INFOCOM, pp.2407-2415,Hong Kong, April, 2015.

[9] L.Deshotels, "Inaudible Sound as a Covert Channel in Mobile Devices," USENIX Workshop for Offensive Technologies, 2014.

[10] Kyu-jin Lee, Dong-ho Cha, Sun-ha Hwang, Kye-san Lee, "Study on Scalable Video Coding Signals Transmisson Schme using LEDID System" Kics, '11-10 Vol.36 No.10 R. Barry, "Wireless Infrared Communication,” Kluwer Academic Press, Boston, MA, 1994. 